\title{
Effect of Variety and Growth Regulator Concentration on Success of Air Layering in Plum
}

\section{Shailendra Nath Mozumder1*, Md. Iqbal Haque1, Md. Masudul Haque ${ }^{1}$, Dristi Sarkar², Muhammad Shahiduzzaman ${ }^{1}$}

\author{
${ }^{1}$ Regional Spices Research Center, Bangladesh Agricultural Research Institute, Gazipur, Bangladesh \\ ${ }^{2}$ Bangladesh Maize and Wheat Research Institute, Nashipur, Bangladesh \\ Email: *shailenbari95@yahoo.com
}

How to cite this paper: Mozumder, S.N., Haque, M.I., Haque, M.M., Sarkar, D. and Shahiduzzaman, M. (2022) Effect of Variety and Growth Regulator Concentration on Success of Air Layering in Plum. Agricultural Sciences, 13, 65-73.

https://doi.org/10.4236/as.2022.131006

Received: July 22, 2021

Accepted: January 24, 2022

Published: January 27, 2022

Copyright ( 2022 by author(s) and Scientific Research Publishing Inc. This work is licensed under the Creative Commons Attribution International License (CC BY 4.0).

http://creativecommons.org/licenses/by/4.0/

\begin{abstract}
The experiment was conducted at the Regional Spices Research Center, BARI, Gazipur during June 2016 to October 2017 to evaluate the effect of genotype and growth regulator (IBA) on the success and performance of plum air layering. Air layering was done on two plum varieties (BARI Alubokhara-1 and PD Gaz 004) using seven levels of IBA concentration (viz. 0, 1000, 2000, $3000,4000,5000$ and $6000 \mathrm{ppm}$ ) under a complete randomized (factorial) design (CRD). Significant variation was observed on rooting, establishment and death of layers due to variety and IBA concentrations except rooting and separation time. The un-fruiting line PD Gaz 004 showed outstanding performance in rooting and survivability of layers over BARI Alubokhara-1. Poor rooting and lower establishment caused very high mortality of layers in BARI Alubokhara-1. Rooting and survivability, number of roots, length of roots and leaf production increased with the increasing levels of IBA concentration up to $5000 \mathrm{ppm}$. The maximum (10 out of 10 ) rooting success of layer with $65.83 \%$ and $59.17 \%$ establishment from PD Gaz004 was obtained when 3000 and 4000 ppm IBA was used, respectively. In BARI Alubokhara-1, the highest rooting success (3.08), establishment rate (29.42\%), number of root (4.28/layer) and root length $(5.08 \mathrm{~cm})$ were recorded with $4000 \mathrm{ppm}$ IBA concentration.
\end{abstract}

\section{Keywords}

Growth Regulator, Layering, Plum, Success

\section{Introduction}

Alubokhara or Plum (Prunus domestica) is a valued species in the family Rosa- 
ceae native to Europe and Asia. Prunus domestica is believed to have originated in the area of the Caucusus and Asia Minor [1]. Plums and their juice contain mild laxatives including phenolic compounds, sorbitol, dietary fiber that are thus common home remedies for constipation [2]. Plums also have a high antioxidant content which retards aging [3]. In Bangladesh, the demands of plum usually meet up by importing from other countries like India, China, Turkey, South Korea and Pakistan [4]. After several years evaluation of various germplasm, one was found superior in respect of adaptability, yield and yield attributes, quality, pest and disease tolerance and released as "BARI Alubokhara-1" from the Spices Research Center of Bangladesh Agricultural Research Institute in 2013 [5]. This variety produces more number of fruits (1221/plant/year) in June-July and yielded $10.29 \mathrm{~kg} /$ plant or $6.43 \mathrm{t} / \mathrm{ha}$. Fruits are attractive dark red colored, sour sweet taste (TSS 10.6\%), medium size $(3 \times 2.74 \mathrm{~cm}, 8.67 \mathrm{~g})$ and globular shape having high nutritive and medicinal properties. This variety performed well in past five years and adopted well in the environment of Bangladesh with low disease and pest infestation [4]. The description of BARI Alubokhara-1 is published in the BARI website http://www.bari.gov.bd/ and broadcasted several times on Bangladesh Television in 2014 to 2016. Huge demands of saplings are observed after mass media broadcasting and website publication. Vegetative propagation is essential to disseminate this variety for increasing its cultivation maintaining genetic purity of the variety. Air layering and cutting are most used and popular means of Alubokhara propagation [6]. In rainy season of Bangladesh, many fruit plants showed good shoot and root growth in cuttings and air layering. Little information is available on successful use of plant growth regulator i.e. Indole-3 butyric acid (IBA) for root initiation in Plums in various countries, but no such reports are available in Bangladesh condition. IBA $1000 \mathrm{mg} \cdot \mathrm{L}^{-1}$ was the best in the rooting of plum cuttings [7]. Vegetative propagation using IBA is eco-friendly and no harmful reports so far are known on use of IBA for cuttings and layering establishment. The alternate way of vegetative propagation is grafting on seedlings of the same species. But Alubokhara seeds showed shy germination and death rate of germinated seedlings are very high. Therefore, the present study was designed for development of a successful vegetative propagation of plum with a view to increase its production and profitability through rapid dissemination of BARI Alubokhara-1. The main objectives of experiment were:

1) Standardization of IBA concentration for air layering propagation techniques of Alubokhara (plum) in the environmental condition of Bangladesh.

2) To increase success of layering using growth regulators and appropriate variety.

\section{Materials and Methods}

The experiment was conducted at the Regional Spices Research Center, BARI, Gazipur located in about $40 \mathrm{~km}$ North to Dhaka city with $23^{\circ} 59^{\prime} 29^{\prime \prime}$ North lati- 
tude and $90^{\circ} 24^{\prime} 50^{\prime \prime}$ East longitude and an elevation of $8.50 \mathrm{~m}$ from the sea level during June 2016 to October 2017. The experiment was conducted using a complete randomized design (factorial) using two factors comprising two plum varieties/lines (BARI Alubokhara-1 and PD Gaz 004) and seven levels of IBA concentration $(0,1000,2000,3000,4000,5000$ and $6000 \mathrm{ppm})$. Soil mixture was prepared with 50\% loamy soil and 50\% well decomposed cow dung and kept for 2 weeks before use for layering. Treatment wise hormone solution was taken with a dropper bottle and applied 3/4 drops on the cut surface (from where bark was removed) of the shoot. No hormone was applied for control treatment. Each replication of a single treatment was consisting of 10 layering shoots and a total of 30 for 3 replications was used for each treatment and tagged properly. A total of 420 layering were done for seven IBA concentrations. The cut portions was surrounded with 150 - $200 \mathrm{~g}$ of moist soil mixture, covered with polythene and tied tightly with jute rope thus called the "stool" or "mount". When a number of roots were established and seen over polythene, the air layering seems suitable to separate from the mother plant. A half cut was given at $2-3 \mathrm{~cm}$ below the cut portion or just below the stool of air layering. After one week the layering was separated by final cut from the previous cut place and extra branches and leaves were trimmed out. The trimmed layering shoot was planted in previously prepared $7 " \times 10^{\prime \prime}$ polythene bag removing the layering polythene and kept one week under shade followed by 2 weeks in partial shade for establishment. When a number of roots and shoots were established, the air layering seems suitable to plant in the field. Data on length and number of roots per layering was counted breaking the stool at time of separation while success of detached layers and number of leaves was counted at 30 days after separation. The sample of 5 layers was broken and the numbers of roots were counted and lengths of roots were measured with a digital slide calipers. After separation from the mother plants, success of detached layers and number of leaves were counted at 30 days of planting in the polythene bag. The data were compiled properly and analyzed statistically by MSTAT and MS-Excel Program and mean comparison was done following Dancan's Multiple Range Test [8].

\section{Results and Discussion}

Both the factors, variety and growth regulator showed different extent of variations in respect of rooting time and success as well as growth of root and shoot in plum layering during two years experimentation.

\subsection{Effect of Variety on Success of Air Layering}

BARI Alubokhara-1 was significantly reluctant in rooting compared to the line PD Gaz 004 for successful layering (Table 1). BARI Alubokhara-1 took more time for rooting (41.15 days) and separation (51.8 days) while PD Gaz-004 showed early rooting (33.9 days) and separation (44.2 days). The maximum number of successful layers (8.77) was recorded in PD Gaz-004 but it was much 
Table 1. Effect of variety on success on rooting time and success of plum layering.

\begin{tabular}{|c|c|c|c|c|c|c|c|c|c|c|c|c|}
\hline \multirow{2}{*}{ Variety } & \multicolumn{3}{|c|}{ Days to rooting } & \multicolumn{3}{|c|}{ Days to cut } & \multicolumn{3}{|c|}{ Successful layers (no) } & \multicolumn{3}{|c|}{$\%$ Dead layers } \\
\hline & 2015 & 2016 & Mean & 2015 & 2016 & Mean & 2015 & 2016 & Mean & 2015 & 2016 & Mean \\
\hline BARI Alubokhara-1 & $37.0 \mathrm{a}$ & $45.3 \mathrm{a}$ & $41.15 \mathrm{a}$ & $46.0 \mathrm{a}$ & $57.6 \mathrm{a}$ & $51.8 \mathrm{a}$ & $2.11 \mathrm{~b}$ & $3.40 \mathrm{~b}$ & $2.76 \mathrm{~b}$ & $78.89 \mathrm{a}$ & $45.14 \mathrm{a}$ & $61.65 \mathrm{a}$ \\
\hline PD GAZ004 & $33.5 b$ & $34.3 \mathrm{~b}$ & $33.90 \mathrm{~b}$ & $42.5 b$ & $45.8 \mathrm{~b}$ & $44.2 \mathrm{~b}$ & $9.53 \mathrm{a}$ & $8.02 \mathrm{a}$ & $8.77 \mathrm{a}$ & $4.72 b$ & $9.30 \mathrm{~b}$ & $7.01 \mathrm{~b}$ \\
\hline Significance level & NS & $* *$ & * & * & $* *$ & * & $* *$ & $* *$ & ** & $* *$ & * & ** \\
\hline CV $(\%)$ & 3.34 & 4.88 & 4.11 & 4.42 & 5.63 & 5.03 & 12.35 & 12.50 & 12.43 & 14.52 & 14.83 & 14.67 \\
\hline
\end{tabular}

Means having same letter(s) or without letter are not significantly different by DMRT. "ns", “*” and "***” means not significant, significant at $5 \%$ and $1 \%$ probability level, respectively.

lower (2.76) in BARI Alubokhara-1 out of 10 layering. The death rate of BARI Alubokhara-1 was very high (61.65\%) while in PD Gaz-004 only 7.01\% layer was died after separation. Early and profuse rooting resulted less death percentage in PD Gaz 004 while delayed and shy rooting caused higher death rate in BARI Alubokhara-1 may be due to the varietal characteristics under Bangladesh environment.

Table 2 showed the varietal effect on rooting, establishment and growth performances of layering. PD Gaz 004 also showed significantly higher establishment rate $(61.38 \%)$ better rooting $(4.38 /$ layer), longer roots $(5.46 \mathrm{~cm})$ and leaf initiation (14.1/plant) compared to BARI Alubokhara-1 which had only $22.26 \%$ success, 3.76 roots/layer, $4.44 \mathrm{~cm}$ root length and 12.1 leaves/layer.

Better root growth resulted higher success rate in PD Gaz-004 might be due to the cumulative deposited energy because this line flowered only but it does not produce any fruits.

One the other hand, BARI Alubokhara-1 had profuse bearing with very good number of fruits which used some photosynthetic energy and food materials during fruit production.

\subsection{Effect of IBA Concentration}

IBA concentration had significant effect on the success of layering (Table 3). Control treatment and lower dose of IBA took more times to initiate roots compared to higher doses of IBA concentration. Days to separation of layers from the mother plant was very close among the concentration though it was significant at $5 \%$ level. The number of successful layer was significantly higher $((\geq 6))$ with the concentration of IBA $2000 \mathrm{ppm}$ to $6000 \mathrm{ppm}$ and it was lower in control (4.29/10). These findings are partially resembled with the findings [9] where the highest rooting percentage in plum was obtained with IBA treatment of cuttings with $2000 \mathrm{mg} \cdot \mathrm{l}^{-1}$ during summer. But these findings are differed in respect of IBA concentration with the report [7] and [10] who obtained the highest success using $1000 \mathrm{ppm}$ IBA in plum cutting and layering. Another findings [11] showed he maximum success (87.5\%) of plum cuttings from 1500 ppm K-IBA solution. 
Table 2. Effect of variety on establishment and growth performance of plum layering.

\begin{tabular}{|c|c|c|c|c|c|c|c|c|c|c|c|c|}
\hline \multirow{2}{*}{ Variety } & \multicolumn{3}{|c|}{ \% Established } & \multicolumn{3}{|c|}{ No. of roots/ayer } & \multicolumn{3}{|c|}{ Length of root $(\mathrm{cm})$} & \multicolumn{3}{|c|}{ Leaves/plant } \\
\hline & 2015 & 2016 & Mean & 2015 & 2016 & Mean & 2015 & 2016 & Mean & 2015 & 2016 & Mean \\
\hline BARI Alubokhara-1 & $15.00 \mathrm{~b}$ & $29.52 b$ & $22.26 b$ & $3.5 b$ & 4.01 & 3.76 & $4.16 \mathrm{~b}$ & 4.72 & $4.44 \mathrm{~b}$ & $10.20 \mathrm{~b}$ & 14.00 & $12.10 \mathrm{~b}$ \\
\hline PD GAZ004 & $51.81 \mathrm{a}$ & $70.95 a$ & $61.38 \mathrm{a}$ & $4.5 \mathrm{a}$ & 4.26 & 4.38 & $5.63 a$ & 5.28 & $5.46 a$ & $13.10 \mathrm{a}$ & 15.00 & $14.10 \mathrm{a}$ \\
\hline Significance level & ** & ** & $* *$ & NS & NS & NS & $* *$ & NS & * & $* *$ & NS & * \\
\hline CV (\%) & 14.52 & 15.23 & 14.88 & 11.21 & 5.34 & 8.28 & 9.82 & 4.38 & 7.01 & 12.87 & 5.40 & 9.14 \\
\hline
\end{tabular}

Means having same letter(s) or without letter are not significantly different by DMRT. "ns", “*” and "**» means not significant, significant at $5 \%$ and $1 \%$ probability level, respectively.

Table 3. Effect of IBA concentration on success of rooting time and plum layering.

\begin{tabular}{|c|c|c|c|c|c|c|c|c|c|c|c|c|}
\hline \multirow{2}{*}{ IBA } & \multicolumn{3}{|c|}{ Days to rooting } & \multicolumn{3}{|c|}{ Days to cut } & \multicolumn{3}{|c|}{ Successful layers (no) } & \multicolumn{3}{|c|}{ \% Dead layers } \\
\hline & 2015 & 2016 & Mean & 2015 & 2016 & Mean & 2015 & 2016 & Mean & 2015 & 2016 & Mean \\
\hline 0 & $37.96 \mathrm{a}$ & $46.79 \mathrm{a}$ & $42.38 \mathrm{a}$ & $46.63 a$ & $59.04 \mathrm{a}$ & $52.84 \mathrm{a}$ & $4.29 \mathrm{~d}$ & $2.96 \mathrm{~d}$ & $3.63 b$ & $57.08 \mathrm{a}$ & $19.40 \mathrm{~b}$ & $38.24 \mathrm{a}$ \\
\hline 1000 & $35.92 \mathrm{~b}$ & $41.00 \mathrm{~b}$ & $38.46 \mathrm{~b}$ & $44.75 b$ & $52.38 \mathrm{a}$ & $48.57 \mathrm{ab}$ & $5.46 \mathrm{c}$ & $4.88 \mathrm{c}$ & $5.17 \mathrm{ab}$ & $45.42 \mathrm{~b}$ & $20.25 b$ & $32.83 b$ \\
\hline 2000 & $35.25 \mathrm{bc}$ & $38.75 \mathrm{c}$ & $37.00 \mathrm{~b}$ & $44.54 b$ & $50.92 \mathrm{a}$ & $47.73 b$ & $6.33 \mathrm{ab}$ & $6.04 b$ & $6.19 a$ & $36.67 d$ & $24.35 \mathrm{ab}$ & $30.51 b$ \\
\hline 3000 & $34.63 \mathrm{~cd}$ & $38.08 \mathrm{c}$ & $36.36 \mathrm{~b}$ & $43.17 \mathrm{c}$ & $50.21 \mathrm{a}$ & $46.69 \mathrm{~b}$ & $6.25 \mathrm{ab}$ & $7.00 \mathrm{a}$ & $6.63 a$ & $37.50 \mathrm{~cd}$ & $28.90 \mathrm{ab}$ & $38.20 \mathrm{a}$ \\
\hline 4000 & $34.17 \mathrm{de}$ & $37.92 \mathrm{c}$ & $36.05 b$ & $43.29 \mathrm{c}$ & $49.79 \mathrm{a}$ & $46.54 b$ & $6.50 \mathrm{a}$ & $6.79 a$ & $6.65 a$ & $35.00 \mathrm{~cd}$ & $30.00 \mathrm{a}$ & $32.50 \mathrm{~b}$ \\
\hline 5000 & $33.91 \mathrm{e}$ & $38.17 \mathrm{c}$ & $36.04 \mathrm{~b}$ & $43.14 \mathrm{c}$ & $49.71 \mathrm{a}$ & $46.43 b$ & $6.29 \mathrm{ab}$ & $6.33 b$ & $6.31 \mathrm{a}$ & $39.10 \mathrm{~cd}$ & $34.85 a$ & $35.96 \mathrm{ab}$ \\
\hline 6000 & $33.75 \mathrm{e}$ & $37.96 \mathrm{c}$ & $35.86 \mathrm{~b}$ & $43.00 \mathrm{c}$ & $49.75 a$ & $46.38 b$ & $6.08 \mathrm{~b}$ & $6.00 \mathrm{~b}$ & $6.04 \mathrm{a}$ & 39.17 & $22.75 b$ & $30.96 \mathrm{~b}$ \\
\hline Significance. & ** & * & * & * & * & * & $* *$ & $* *$ & * & ** & * & * \\
\hline CV (\%) & 3.34 & 4.88 & 4.11 & 4.42 & 5.63 & 5.03 & 12.35 & 12.50 & 12.43 & 14.52 & 14.83 & 14.68 \\
\hline
\end{tabular}

Means having same letter(s) or without letter are not significantly different by DMRT. "ns", “*” and "**” means not significant, significant at $5 \%$ and $1 \%$ probability level, respectively.

There was significant variation on number of dead layers after separation from the mother plant, rooting and leaf production with various levels of IBA treatment. Indole-butyric-acid (IBA) enhanced root development and root growth by enhancing cell division resulted the maximum success of layering compared to control and lower level of IBA concentration [4].

IBA concentration significantly affected on the establishment and growth of plum layering (Table 4). Higher concentration of IBA resulted more number $(\geq 4)$ of longer $(\geq 5 \mathrm{~cm}$ ) roots compared to control and lower concentration of IBA. Number of leaves was increased with increasing IBA concentration up to $4000 \mathrm{ppm}$.

The highest number of leaves (13.89/layer) was recorded from the application of $4000 \mathrm{ppm}$ IBA and the lowest (11.07/layer) was found from control. IBA helps to accelerate cell division and root initiation in upper parts of the cut portion of the plant resulted more rooting and leaves with higher doses of IBA. Previous report [11] showed that growth regulator enhanced rooting and germination 
Table 4. Effect of IBA concentration on establishment and growth of plum layering.

\begin{tabular}{cccccccccccccccc}
\hline & \multicolumn{3}{c}{ \% Established } & \multicolumn{3}{c}{ No. of roots/layer } & \multicolumn{2}{c}{ Length of root $(\mathrm{cm})$} & \multicolumn{3}{c}{ Leaves/plant } \\
\cline { 2 - 13 } IBA & 2015 & 2016 & Mean & 2015 & 2016 & Mean & 2015 & 2016 & Mean & 2015 & 2016 & Mean \\
\hline 0 & $17.50 \mathrm{~d}$ & $25.00 \mathrm{~d}$ & $21.25 \mathrm{c}$ & $3.36 \mathrm{c}$ & $3.83 \mathrm{~d}$ & $3.60 \mathrm{~b}$ & $3.98 \mathrm{~b}$ & $4.59 \mathrm{~d}$ & $4.29 \mathrm{~b}$ & $10.29 \mathrm{c}$ & $11.84 \mathrm{~d}$ & $11.07 \mathrm{~b}$ \\
1000 & $27.90 \mathrm{c}$ & $43.33 \mathrm{c}$ & $35.62 \mathrm{~b}$ & $3.71 \mathrm{bc}$ & $4.02 \mathrm{c}$ & $3.87 \mathrm{ab}$ & $4.47 \mathrm{~b}$ & $4.86 \mathrm{c}$ & $4.67 \mathrm{ab}$ & $11.03 \mathrm{bc}$ & $14.15 \mathrm{c}$ & $12.59 \mathrm{ab}$ \\
2000 & $37.50 \mathrm{ab}$ & $55.00 \mathrm{~b}$ & $46.25 \mathrm{a}$ & $4.30 \mathrm{a}$ & $4.11 \mathrm{bc}$ & $4.21 \mathrm{a}$ & $5.25 \mathrm{a}$ & $5.02 \mathrm{~b}$ & $5.14 \mathrm{a}$ & $12.71 \mathrm{a}$ & $14.81 \mathrm{~b}$ & $13.76 \mathrm{a}$ \\
3000 & $42.10 \mathrm{a}$ & $61.25 \mathrm{a}$ & $51.68 \mathrm{a}$ & $4.27 \mathrm{a}$ & $4.25 \mathrm{a}$ & $4.26 \mathrm{a}$ & $5.19 \mathrm{a}$ & $5.14 \mathrm{ab}$ & $5.17 \mathrm{a}$ & $11.95 \mathrm{abc}$ & $14.90 \mathrm{ab}$ & $13.43 \mathrm{a}$ \\
4000 & $41.30 \mathrm{a}$ & $60.42 \mathrm{a}$ & $50.86 \mathrm{a}$ & $4.46 \mathrm{a}$ & $4.29 \mathrm{a}$ & $4.38 \mathrm{a}$ & $5.43 \mathrm{a}$ & $5.134 \mathrm{ab}$ & $5.28 \mathrm{a}$ & $12.57 \mathrm{ab}$ & $15.21 \mathrm{ab}$ & $13.89 \mathrm{a}$ \\
5000 & $37.60 \mathrm{~b}$ & $54.58 \mathrm{~b}$ & $46.09 \mathrm{a}$ & $4.25 \mathrm{a}$ & $4.28 \mathrm{a}$ & $4.27 \mathrm{a}$ & $5.24 \mathrm{a}$ & $5.174 \mathrm{a}$ & $5.21 \mathrm{a}$ & $11.97 \mathrm{abc}$ & $15.37 \mathrm{a}$ & $13.67 \mathrm{a}$ \\
6000 & $34.20 \mathrm{~b}$ & $52.08 \mathrm{~b}$ & $43.14 \mathrm{ab}$ & $4.03 \mathrm{ab}$ & $4.19 \mathrm{ab}$ & $4.11 \mathrm{a}$ & $5.05 \mathrm{a}$ & $5.10 \mathrm{ab}$ & $5.08 \mathrm{a}$ & $11.27 \mathrm{abc}$ & $11.90 \mathrm{f}$ & $11.59 \mathrm{c}$ \\
\hline Significance & $* *$ & $* *$ & $* *$ & $* *$ & $* *$ & $* *$ & $* *$ & $* *$ & $* *$ & $* *$ & $* *$ & $*$ \\
CV (\%) & 14.52 & 15.23 & 14.88 & 11.21 & 5.34 & 8.28 & 9.82 & 4.38 & 7.10 & 12.87 & 5.40 & 9.14 \\
\hline
\end{tabular}

Means having same letter(s) or without letter are not significantly different by DMRT. "ns”, “*” and "**» means not significant, significant at $5 \%$ and $1 \%$ probability level, respectively.

increasing $\alpha$-amylase activities in the seeds and plant.

\subsection{Combined Effect of Variety and IBA Concentration}

Both the variety and line showed incremental rate of rooting and success of layers with increasing IBA concentration up to a certain level (Table 5). BARI Alubokhara-1 took the maximum time for rooting (46.5 days) and detachment (57.1 days) of layers in control treatment (without IBA). Early rooting (32.5 days) and cut time (42.8 days) was observed in PD Gaz 004 with 6000 ppm IBA application. The maximum (9.59 out of 10 ) rooting success of layer was obtained from PD Gaz 004 with 3000 IBA concentration while it was very poor $(0.96 / 10)$ in BARI Alubokhara-1 in control. The Highest percentage of dead layers (71\%) was recorded in BARI Alubokhara-1 with 3000 ppm IBA and it was the lowest (3.35\%) in PD Gaz 004 with 2000 ppm IBA treatment.

Establishment rate and rooting significantly affected with the combined effect of variety and IBA but number of leaves was similar (Table 6). The highest establishment rate (73.33\%) was recorded in PD Gaz 004 with 3000 ppm IBA but it was only $5.42 \%$ in BARI Alubokhara-1 without IBA. The line PD Gaz 004 showed better rooting ( 4.58 roots/layer) root length $(5.68 \mathrm{~cm})$ and leaf production (14.60/layer) with higher doses $(5000-6000 \mathrm{ppm})$ of growth regulator (IBA).

The number of leaves per layer at 30 days after separation showed insignificant variation with different level of IBA or varieties. Among BARI Alubokhara-1, the highest establishment rate $(34.29 .42 \%)$, number of roots (4.21/layer) and root length $(4.99 \mathrm{~cm})$ and leaves (13.30/layer) was recorded with $4000 \mathrm{ppm}$ solution. 
Table 5. Combined effect of variety and IBA concentration on success of plum layering.

\begin{tabular}{|c|c|c|c|c|c|c|c|c|c|c|c|}
\hline \multirow{2}{*}{ Var. } & \multirow{2}{*}{ IBA } & \multicolumn{3}{|c|}{ Days to rooting } & \multirow{2}{*}{$\begin{array}{c}\text { Days to } \\
\text { cut }\end{array}$} & \multicolumn{3}{|c|}{ Successful layers (no) } & \multicolumn{3}{|c|}{ \% Dead layers } \\
\hline & & 2015 & 2016 & Mean & & 2015 & 2016 & Mean & 2015 & 2016 & Mean \\
\hline \multirow{7}{*}{ 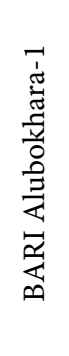 } & 0 & 39.9 & $53.0 \mathrm{a}$ & $46.5 \mathrm{a}$ & 57.1 & $0.83 \mathrm{f}$ & $1.08 \mathrm{~g}$ & $0.96 \mathrm{~d}$ & $91.67 \mathrm{a}$ & $33.0 \mathrm{c}$ & $62.34 \mathrm{~b}$ \\
\hline & 1000 & 37.8 & $46.9 \mathrm{ab}$ & $42.4 \mathrm{a}$ & 52.8 & $1.42 \mathrm{f}$ & $2.58 \mathrm{f}$ & $2.00 \mathrm{~cd}$ & $85.83 a$ & $33.0 \mathrm{c}$ & $59.42 b$ \\
\hline & 2000 & 37.0 & $44.3 \mathrm{ab}$ & $40.7 \mathrm{ab}$ & 51.9 & $2.67 \mathrm{c}$ & $3.58 \mathrm{e}$ & $3.13 c$ & $73.33 c$ & $42.0 \mathrm{c}$ & $57.67 \mathrm{~b}$ \\
\hline & 3000 & 36.3 & $43.3 \mathrm{~b}$ & $39.8 \mathrm{ab}$ & 50.2 & $2.50 \mathrm{de}$ & $4.83 \mathrm{~d}$ & $3.67 \mathrm{c}$ & $75.00 \mathrm{bc}$ & $67.0 \mathrm{~b}$ & $71.00 \mathrm{a}$ \\
\hline & 4000 & 35.8 & $43.1 \mathrm{~b}$ & $39.5 \mathrm{ab}$ & 50.1 & $3.08 \mathrm{~cd}$ & $4.42 \mathrm{~d}$ & $3.75 \mathrm{c}$ & $69.17 \mathrm{~cd}$ & $50.0 \mathrm{bc}$ & $59.59 \mathrm{~b}$ \\
\hline & 5000 & 35.6 & $43.5 b$ & $39.6 \mathrm{ab}$ & 50.0 & 2.63de & $3.83 \mathrm{e}$ & $3.23 c$ & $73.75 \mathrm{bc}$ & $58.0 \mathrm{bc}$ & $65.88 \mathrm{ab}$ \\
\hline & 6000 & 35.4 & $43.2 \mathrm{~b}$ & $39.3 \mathrm{ab}$ & 50.0 & $2.17 \mathrm{e}$ & $3.50 \mathrm{e}$ & $2.84 \mathrm{~cd}$ & $78.33 b$ & $33.0 \mathrm{c}$ & $55.67 \mathrm{~b}$ \\
\hline \multirow{7}{*}{ 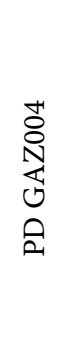 } & 0 & 36.0 & $40.6 \mathrm{bc}$ & $38.3 \mathrm{ab}$ & 48.6 & $7.75 b$ & $4.83 \mathrm{~d}$ & $6.29 b$ & $22.50 \mathrm{e}$ & $5.80 \mathrm{bc}$ & $14.15 \mathrm{c}$ \\
\hline & 1000 & 34.0 & $35.1 \mathrm{c}$ & $34.6 \mathrm{~b}$ & 44.4 & $9.50 \mathrm{a}$ & $7.17 \mathrm{c}$ & $8.34 \mathrm{ab}$ & $5.00 \mathrm{f}$ & $7.50 \mathrm{~b}$ & $6.25 \mathrm{~d}$ \\
\hline & 2000 & 33.5 & $33.3 c$ & $33.4 \mathrm{~b}$ & 43.6 & $10.0 \mathrm{a}$ & $8.50 \mathrm{~b}$ & $9.25 a$ & $0.00 \mathrm{f}$ & $6.70 \mathrm{~b}$ & $3.35 \mathrm{~d}$ \\
\hline & 3000 & 33.0 & $32.9 c$ & $32.9 b$ & 43.2 & $10.0 \mathrm{a}$ & $9.17 \mathrm{a}$ & $9.59 a$ & $0.00 \mathrm{f}$ & $10.80 \mathrm{ab}$ & $5.40 \mathrm{~d}$ \\
\hline & 4000 & 32.6 & $32.8 \mathrm{c}$ & $32.7 \mathrm{~b}$ & 43.0 & $9.92 \mathrm{a}$ & $9.17 \mathrm{a}$ & $9.55 a$ & $0.83 \mathrm{f}$ & $10.00 \mathrm{ab}$ & $5.42 \mathrm{~d}$ \\
\hline & 5000 & 32.3 & $32.8 \mathrm{c}$ & $32.6 b$ & 42.8 & $9.96 a$ & $8.83 \mathrm{ab}$ & $9.39 a$ & $0.40 \mathrm{f}$ & $11.70 \mathrm{a}$ & $6.05 \mathrm{~d}$ \\
\hline & 6000 & 32.1 & $32.8 \mathrm{c}$ & $32.5 b$ & 42.8 & $10.00 \mathrm{a}$ & $8.50 \mathrm{~b}$ & $9.25 \mathrm{a}$ & $0.00 \mathrm{f}$ & $12.50 \mathrm{a}$ & $6.25 \mathrm{~d}$ \\
\hline \multicolumn{2}{|c|}{ Significance. } & NS & * & $*$ & NS & $* *$ & * & * & $* *$ & * & * \\
\hline \multicolumn{2}{|c|}{ CV (\%) } & 3.34 & 4.88 & 4.11 & 5.03 & 12.35 & 12.50 & 12.43 & 14.52 & 14.83 & 14.68 \\
\hline
\end{tabular}

Means having same letter(s) or without letter are not significantly different by DMRT. "ns", “*” and “**” means not significant, significant at $5 \%$ and $1 \%$ probability level, respectively.

Table 6. Combined effect of variety and IBA concentration on establishment and growth of plum layering.

\begin{tabular}{|c|c|c|c|c|c|c|c|c|c|c|c|}
\hline \multirow{2}{*}{ Variety } & \multirow{2}{*}{ IBA } & \multicolumn{3}{|c|}{$\%$ Established } & \multicolumn{3}{|c|}{ No. of roots /layer } & \multicolumn{3}{|c|}{ Length of root $(\mathrm{cm})$} & \multirow{2}{*}{$\begin{array}{c}\text { Leaves/ } \\
\text { plant }\end{array}$} \\
\hline & & 2015 & 2016 & Mean & 2015 & 2016 & Mean & 2015 & 2016 & Mean & \\
\hline \multirow{7}{*}{ 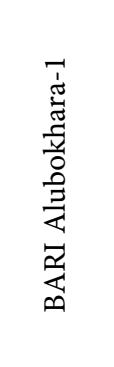 } & 0 & $3.33 \mathrm{~g}$ & $7.50 \mathrm{~d}$ & $5.42 \mathrm{~h}$ & $2.52 \mathrm{e}$ & 3.69 & $3.11 \mathrm{~b}$ & $2.78 \mathrm{e}$ & 4.24 & $3.51 b$ & 9.70 \\
\hline & 1000 & $9.17 \mathrm{~g}$ & $22.50 \mathrm{~d}$ & $15.84 \mathrm{~g}$ & 3.08de & 3.90 & $3.49 \mathrm{ab}$ & $3.36 \mathrm{e}$ & 4.57 & $3.97 \mathrm{~b}$ & 11.37 \\
\hline & 2000 & $18.33 \mathrm{f}$ & $31.67 \mathrm{~cd}$ & $25.00 \mathrm{f}$ & $4.13 \mathrm{ab}$ & 4.02 & $4.08 \mathrm{a}$ & $4.85 \mathrm{bcd}$ & 4.77 & $4.81 \mathrm{ab}$ & 13.23 \\
\hline & 3000 & $18.33 \mathrm{f}$ & $41.67 \mathrm{bcd}$ & 30.00de & $3.88 \mathrm{bc}$ & 4.13 & $4.01 \mathrm{a}$ & $4.64 \mathrm{~cd}$ & 4.89 & $4.77 \mathrm{ab}$ & 12.65 \\
\hline & 4000 & $29.42 \mathrm{f}$ & $39.17 \mathrm{bcd}$ & $34.29 \mathrm{~d}$ & $4.28 \mathrm{ab}$ & 4.13 & $4.21 \mathrm{a}$ & $5.09 \mathrm{a}-\mathrm{d}$ & 4.89 & $4.99 \mathrm{ab}$ & 13.30 \\
\hline & 5000 & $23.33 \mathrm{f}$ & $32.50 \mathrm{~cd}$ & $27.92 \mathrm{e}$ & $3.80 \mathrm{bc}$ & 4.10 & $3.95 \mathrm{ab}$ & $4.67 \mathrm{~cd}$ & 4.84 & $4.76 \mathrm{ab}$ & 12.70 \\
\hline & 6000 & $17.50 \mathrm{f}$ & $31.67 \mathrm{~cd}$ & $24.59 \mathrm{ef}$ & $3.31 \mathrm{~cd}$ & 4.13 & $3.72 \mathrm{ab}$ & $4.25 \mathrm{~d}$ & 4.83 & $4.54 \mathrm{ab}$ & 11.85 \\
\hline \multirow{7}{*}{ 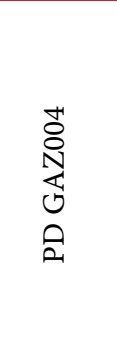 } & 0 & $31.67 \mathrm{e}$ & $42.50 \mathrm{bcd}$ & $37.09 \mathrm{~d}$ & $4.21 \mathrm{ab}$ & 3.96 & $4.09 \mathrm{a}$ & $5.18 \mathrm{abc}$ & 4.95 & $5.07 \mathrm{ab}$ & 12.40 \\
\hline & 1000 & $46.67 \mathrm{~d}$ & $64.17 \mathrm{abc}$ & $55.42 \mathrm{c}$ & $4.34 \mathrm{ab}$ & 4.13 & $4.24 \mathrm{a}$ & $5.57 \mathrm{ab}$ & 5.14 & $5.36 \mathrm{a}$ & 13.80 \\
\hline & 2000 & $56.67 \mathrm{bc}$ & $78.33 \mathrm{a}$ & $67.50 \mathrm{ab}$ & $4.48 \mathrm{ab}$ & 4.20 & $4.34 \mathrm{a}$ & $5.65 \mathrm{ab}$ & 5.26 & $5.46 \mathrm{a}$ & 14.40 \\
\hline & 3000 & $65.83 a$ & $80.83 a$ & $73.33 \mathrm{a}$ & $4.65 \mathrm{ab}$ & 4.36 & $4.51 \mathrm{a}$ & $5.75 \mathrm{ab}$ & 5.38 & $5.57 \mathrm{a}$ & 14.18 \\
\hline & 4000 & $59.17 \mathrm{ab}$ & $81.67 \mathrm{a}$ & $70.42 \mathrm{a}$ & $4.64 \mathrm{ab}$ & 4.45 & $4.55 \mathrm{a}$ & $5.76 \mathrm{ab}$ & 5.37 & $5.57 \mathrm{a}$ & 14.48 \\
\hline & 5000 & $55.00 \mathrm{bc}$ & $76.67 \mathrm{a}$ & $65.84 \mathrm{ab}$ & $4.68 \mathrm{a}$ & 4.47 & $4.58 \mathrm{a}$ & $5.85 a$ & 5.50 & $5.68 \mathrm{a}$ & 14.55 \\
\hline & 6000 & $50.83 \mathrm{~cd}$ & $72.50 \mathrm{ab}$ & $61.67 \mathrm{~b}$ & $4.74 \mathrm{a}$ & 4.25 & $4.50 \mathrm{a}$ & $5.86 a$ & 5.37 & $5.62 \mathrm{a}$ & 14.60 \\
\hline \multicolumn{2}{|c|}{ Significance } & $* *$ & * & * & $* *$ & NS & * & $* *$ & NS & * & NS \\
\hline \multicolumn{2}{|c|}{ CV (\%) } & 14.52 & 15.23 & 14.88 & 11.21 & 5.34 & 8.28 & 9.82 & 4.38 & 7.10 & 9.14 \\
\hline
\end{tabular}

Means having same letter(s) or without letter are not significantly different by DMRT. "ns", “*” and “**” means not significant, significant at $5 \%$ and $1 \%$ probability level, respectively. 
IBA accelerates cell division and root initiation at high humidity and temperature resulted more rooting and leaves with higher doses of IBA in layering. The result from this observation was partially resembled with some findings such as [9] gets maximum success with 2000 ppm IBA. Another researcher [11] obtained from 1500 ppm IBA while [9] and [7] got the maximum success with 1000 ppm IBA concentration. All the findings were varied because those experiments were conducted in different environment, soils, climates and times. These findings are resembled with the findings [12] observed versatile variation of plums rooting with various weather and chemical treatments. Saplings produced from layering of the un-fruiting line PD Gaz-004 is kept for cleft grafting of BARI Alubokhara-1 scions in next season for the production of grafted saplings of BARI Alubokhara-1.

\section{Conclusion}

Variety and IBA concentration significantly influenced on the success and rooting of layers under Bangladesh condition. Plum layering using 3000 to 4000 ppm IBA solution seems better for successful vegetative propagation in Bangladesh.

\section{Conflicts of Interest}

The authors declare no conflicts of interest regarding the publication of this paper.

\section{References}

[1] Eryomine, G.V. (2013) New Data on Origin of Prunus domestica L. International Symposium on Plum and Prune Genetics, Breeding and Pomology, 283, 4.

[2] Miletić, N., Popović, B., Mitrović, O. and Kandić, M. (2012) Phenolic Content and Antioxidant Capacity of Fruits of Plum cv. "Stanley" (Prunus domestica L.) as Influenced by Maturity Stage and On-Tree Ripening. AJCS, 6, 681-687.

[3] Stacewicz, S.M., Bowen, P.E., Hussain, E.A., Wood, B.I.D. and Farnsworth, N.R. (2001) Chemical Composition and Potential Health Effects of Prunes: A Functional Food. Critical Reviews in Food Science and Nutrition, 41, 251-286. https://doi.org/10.1080/20014091091814

[4] Mozumder, S.N., Hoque, M.I., Akter, S. and Banik, B.R. (2014) Bangladeshe Alubokhara chash (Booklet in Bengali = Cultivation of plum in Bangladesh). BARI Agro. techbklt no. 04/2014., 1-14.

[5] Anonymous (2014) Annual Report 2013-14. Spices Research Center, BARI, Shibganj, Bogra, p. 169.

[6] Food \& Agriculture Organization (FAO) (2011) The State of Food and Agriculture 2010-2011. Rome, Italy.

[7] Neto M., Telles, U.R., Allan, C. and Antonio, B.L. (2006) Rooting of Hardwood Cuttings from Plum Treated with Indol Butiric Acid. Ciência Rural, 36, 448-452. https://doi.org/10.1590/S0103-84782006000200014

[8] Zaman, S.M.H., Rahim, K. and Hawlader, M. (1987) Simple Lessons from Biometry. Bangladesh Rice Research Institute, Gazipur, 29-34.

[9] Sharma, S.D. and Aier, N.B. (2002) Seasonal Rooting Behaviour of Cuttings of Plum 
Cultivars as Influenced by IBA Treatments. Scientia Horticulturae, 40, 297-303. https://doi.org/10.1016/0304-4238(89)90103-9

[10] Canli, F.A. and Sefer, B. (2009) Effects of Indolebutyric Acid on Adventitious Root Formation from Semi-Hardwood Cuttings of "Sarierik" Plum. Journal of Applied Biosciences, 3, 45-48.

[11] Andrea, L.S. and Dirr, M.A. (1996) Timing and K-IBA Treatments Affect Rooting of Stem Cuttings of Koch (Japanese Plum). HortScience, 31, 222-223. https://doi.org/10.21273/HORTSCI.31.2.222

[12] Nacheva, L., Nacheva, A., Rankova, Z., Gercheva, P. and Zlatev, Z. (2012) Stress Responses of the Cherry Dwarf Rootstock Gisela 5-Prunus cerasus L. $\times$ Prunus canescens L. Fruit Growing Institute, Agricultural University, Plovdiv. 